\title{
RGC32 deficiency protects against high-fat diet-induced obesity and insulin resistance in mice
}

\author{
Xiao-Bing Cui', Jun-Na Luan', Jianping $\mathrm{Ye}^{3}$ and Shi-You Chen ${ }^{1,2}$ \\ ${ }^{1}$ Department of Physiology and Pharmacology, University of Georgia, 501 D.W. Brooks Drive, Athens, \\ Georgia 30602, USA \\ ${ }^{2}$ Renmin Hospital, Hubei University of Medicine, Shiyan, Hubei 442000, China \\ ${ }^{3}$ Antioxidant and Gene Regulation Laboratory, Pennington Biomedical Research Center, Louisiana State University \\ System, Baton Rouge, Louisiana, USA
}

Correspondence should be addressed to $S$-Y Chen

Email sc229@uga.edu

\begin{abstract}
Obesity is an important independent risk factor for type 2 diabetes, cardiovascular diseases and many other chronic diseases. Adipose tissue inflammation is a critical link between obesity and insulin resistance and type 2 diabetes and a contributor to disease susceptibility and progression. The objective of this study was to determine the role of response gene to complement 32 (RGC32) in the development of obesity and insulin resistance. WT and RGC32 knockout $\left(R_{c c 32} 2^{-1}(R g c c)\right)$ mice were fed normal chow or high-fat diet (HFD) for 12 weeks. Metabolic, biochemical, and histologic analyses were performed. 3T3-L1 preadipocytes were used to study the role of RGC32 in adipocytes in vitro. Rgc32 ${ }^{-1-}$ mice fed with HFD exhibited a lean phenotype with reduced epididymal fat weight compared with WT controls. Blood biochemical analysis and insulin tolerance test showed that RGC32 deficiency improved HFD-induced dyslipidemia and insulin resistance. Although it had no effect on adipocyte differentiation, RGC32 deficiency ameliorated adipose tissue and systemic inflammation. Moreover, $R g C 32^{-1-}$ induced browning of adipose tissues and increased energy expenditure. Our data indicated that RGC32 plays an important role in diet-induced obesity and insulin resistance, and thus it may serve as a potential novel drug target for developing therapeutics to treat obesity and metabolic disorders.
\end{abstract}

\author{
Key Words \\ - response gene to \\ complement 32 \\ - obesity \\ - insulin resistance \\ - adipose tissue
}

\section{Introduction}

Obesity is an important independent risk factor for type 2 diabetes and cardiovascular diseases, and it is associated with high morbidity and mortality (Friedman 2003). Although a range of therapies have been developed, these pharmaceutical therapies often have adverse side effects or limited efficacy (Bandyopadhyay 2009, Distefano \& Watanabe 2010). Therefore, a continued effort is required to identify novel drug targets and develop more effective therapeutics for the treatment of obesity.
Adipose tissue plays a critical role in energy homeostasis, not only in storing triglycerides but also in secreting hormones and biologically active molecules that regulate cellular lipid storage capacity, tissue and systemic insulin sensitivity, and metabolic energy balance (Chatterjee et al. 2014). Although the underlying mechanism is not fully understood, adipose tissue inflammation has been acknowledged as a critical link between obesity and insulin resistance and type 2 diabetes, and as a contributor

Published by Bioscientifica Ltd. 
to disease susceptibility and progression (Mori et al. 2010). Adipose tissue inflammation, characterized by increased secretion of proinflammatory cytokines and chemokines including interleukin 6 (IL6), tumor necrosis factor $\alpha(T N F \alpha)$, and IL12, contributes to low-grade systemic inflammation, insulin resistance, and metabolic disorders (Xu et al. 2003, Hotamisligil 2006). Blocking the function of proinflammatory cytokines or chemokines results in improved insulin sensitivity and glucose homeostasis (Uysal et al. 1997, Kanda et al. 2006). Therefore, genes or molecules that control obesity or adipose tissue inflammation are promising therapeutic targets for insulin resistance, type 2 diabetes, and cardiovascular complications.

Response gene to complement 32 (RGC32) is expressed in numerous human organs and tissues including placenta, kidney, liver, heart, and brain (Badea et al. 1998). Functionally, RGC32 plays an important role in cell proliferation, differentiation (Li et al. 2007, Fosbrink et al. 2009, Wang et al. 2011), fibrosis (Li et al. 2011a), and cancer (Fosbrink et al. 2005, Vlaicu et al. 2010, Kim et al. 2011). Recently, we have generated RGC32 knockout $\left(R g c 32^{-/-}(R g C c)\right)$ mice and found that $R g c 32^{-/-}$mice were born smaller than their WT littermates because of the impaired placental angiogenesis (Cui et al. 2013). However, it is unknown if RGC32 plays a role in postnatal metabolism. In this study, we demonstrate that high-fat diet (HFD) dramatically induces RGC32 expression in the adipose tissue. RGC32 deficiency attenuates HFD-induced obesity and insulin resistance in mice. The beneficial effect of RGC32 deficiency is due to the decreased adipose tissue content and systemic inflammation and increased energy expenditure of adipose tissue. This is the first report showing that RGC32 deficiency prevents diet-induced obesity and insulin resistance in mice. Therefore, RGC32 may serve as a potential novel drug target for preventing obesity and type 2 diabetes.

\section{Materials and methods}

\section{Animals and diets}

$R g c 32^{-/-}$mice on the C57BL/6 background were generated and genotyped as described previously (Cui et al. 2013). The parallel line WT C57BL/6 mice were purchased from the Jackson Laboratory (Bar Harbor, ME, USA). The agematched WT and $\mathrm{RgC} \mathrm{2}^{-/-}$male mice were maintained on normal chow for 8 weeks, after which they were fed with either normal chow (25\% protein, $62 \%$ carbohydrate, and $13 \%$ fat; $3.07 \mathrm{kcal} / \mathrm{g} ; 5053$, LabDiet, St. Louis, MO, USA) or
HFD (20\% protein, 40\% carbohydrate, and 40\% fat; $4.5 \mathrm{kcal} / \mathrm{g}$; D12108C, Research Diets, New Brunswick, NJ, USA) for an additional 12 weeks. The mice were fasted overnight and anesthetized (2.0\% isoflurane), and blood was collected by direct cardiac puncture. Epididymal fat was carefully removed and weighed. A portion of the epididymal fat was fixed in $4 \%$ paraformaldehyde for histological analysis, whereas the other portion was stored at $-80^{\circ} \mathrm{C}$ for RNA and protein preparation. All animals were housed under conventional conditions in the animal care facilities and received humane care in compliance with the Principles of Laboratory Animal Care formulated by the National Society for Medical Research and the Guide for the Care and Use of Laboratory Animals. All experimental procedures were approved by Institutional Animal Care and Use Committee (IACUC) of University of Georgia.

\section{Body weight and metabolic studies}

Mice were weighed every 4 weeks. For metabolic studies, the mice were housed individually in metabolic cages (model 3600M021, Techniplast, Buguggiate, VA, Italy) with free access to food and water. After a 5-day period of acclimatization, daily food and water intakes were recorded, and fecal pellets and urine were weighed. The mice were observed for 3 days in the metabolic cage and then were put back in their original cages. To assess the energy expenditure, the mice were fasted overnight and weighed. This body weight was used as a baseline. The mice were fasted for an additional $8 \mathrm{~h}$ and weighed, and then the percentage change in the body weight from the baseline was calculated.

\section{Glucose and insulin tolerance tests}

For the glucose tolerance test (GTT), mice were fasted overnight followed by an i.p. glucose injection $(1 \mathrm{~g} / \mathrm{kg}$ body weight). Blood glucose was measured by tail bleeding using the One-Touch AccuChek Glucometer (Roche) at indicated times. For the insulin tolerance test (ITT), mice without fast were injected intraperitoneally with insulin (Sigma-Aldrich) at a dose of $1.5 \mathrm{IU} / \mathrm{kg}$ body weight, and blood glucose was measured at indicated times.

\section{Blood biochemical analysis}

Serum samples were analyzed for adiponectin, leptin, insulin, triglyceride, and cholesterol using the Adiponectin Mouse ELISA Kit (Abcam, Cambridge, MA, USA), Leptin Mouse ELISA Kit (Abcam), Rat/Mouse Insulin ELISA Kit (Millipore, Billerica, MA, USA), Triglyceride Quantification

Published by Bioscientifica Ltd 
Kit (Abcam), and HDL and LDL/VLDL Cholesterol Assay Kit (Abcam).

\section{Cytometric bead array immunoassay}

Serum from individual mouse was assayed for inflammatory markers using the Cytometric Bead Array Mouse Inflammation Kit (BD Biosciences, San Jose, CA, USA). Data were collected by FACSCalibur flow cytometer (Becton Dickinson, Franklin Lakes, NJ, USA).

\section{T3-L1 cell culture and differentiation}

3T3-L1 preadipocytes (ATCC, Manassas, VA, USA) were maintained in DMEM (Invitrogen) supplemented with 10\% FBS (Sigma). At confluence, adipocyte differentiation was induced by treatment with $1 \mu \mathrm{mol} / \mathrm{l}$ dexamethasone (Sigma), $500 \mu \mathrm{mol} / 1$ isobutylmethylxanthine (Sigma), $10 \mu \mathrm{mol} / \mathrm{l}$ pioglitazone, and $100 \mathrm{nmol} / \mathrm{l}$ insulin (Sigma) for 2 days. The cells were then incubated in $100 \mathrm{nmol} / \mathrm{l}$ insulin-containing medium for 6 days to induce lipid accumulation. The medium was replaced every other day.

\section{Oil Red O staining}

Culture plates were washed by PBS, and cells were fixed in $4 \%$ formaldehyde for $1 \mathrm{~h}$, followed by staining with Oil Red O (Sigma) for $1 \mathrm{~h}$. Oil Red O was prepared by diluting a stock solution (0.5\% in isopropanol) with water $(60: 40$ $\mathrm{vol} / \mathrm{vol}$ ) followed by filtration. After staining, the plates were washed twice in water and photographed. The dye was then extracted with $100 \%$ isopropanol, and the absorbance was determined at $520 \mathrm{~nm}$.

\section{RNA extraction and real-time quantitative RT-PCR}

Quantitative RT-PCR (qPCR) was performed in a Mx3005P qPCR machine using SYBR Green master mix (Agilent Technologies, Santa Clara, CA, USA) as described previously (Li et al. 2011b). Each sample was amplified in triplicate. The expression of each gene was normalized with cyclophilin. Primer sequences are summarized in Table 1.

\section{Western blotting analysis}

Western blotting was performed as described previously (Cui et al. 2012). Antibodies against hormone-sensitive lipase (LIPE, Cell Signaling Technology, 4107S, Danvers, MA, USA), peroxisome proliferator-activated receptor $\alpha$ (PPAR $\alpha)$, (Abcam, ab8934), PPAR gamma coactivator $1 \alpha$ (PGC1 $\alpha)$
Table 1 Primer sequences utilized for qPCR evaluation of gene expression

Gene

Primer sequence

$\operatorname{Rgc32}$

F: 5'-GTG GTC TTT GGG AAG GTG AA-3'

R: 5'-TTA CAG GAC ATT GCG AGC AG-3'

F: $5^{\prime}-$ CCG ATC TGG ACA GGA CCT TA-3'

Ppary

R: 5'-AGC TTC ACT CTC CGA ACT GC-3'

F: $5^{\prime}$-ATCCCTGGTTTCATTAACCT-3'

R: 5'-GCTCCATAAAGTCACCAAAG-3'

Ppar $\alpha$

F: 5'-GGATGTCACACAATGCAATTCG-3'

R: $5^{\prime}$-TCACAGAACGGCTTCCTCAGGT-3'

$C / E b p \alpha$

F: $5^{\prime}$-CAAGAACAGCAACGAGTACCG-3'

R: $5^{\prime}$-GTCACTGGTCAACTCCAGCAC- $3^{\prime}$

Adiponectin

F: $5^{\prime}$-TGTTCCTCTTAATCCTGCCCA-3'

R: 5'-CCAACCTGCACAAGTTCCCTT- ${ }^{\prime}$

Leptin

F: 5'-GTGGCTTTGGTCCTATCTGTC-3'

R: 5'-CGTGTGTGAAATGTCATTGATCC-3'

Lipe

F: $5^{\prime}$-AAGGACTTGAGCAACTCAGA-3'

R: $5^{\prime}$-TTGACTATGGGTGACGTGTA-3'

Pgc1 $\alpha$

F: 5'-ATACCGCAAAGAGCACGAGAA-3'

R: 5'-CTCAAGAGCAGCGAAAGCGTCACA-3'

$1 / 6$

F: 5'-CTGCAAGAGACTTCCATCCAG-3'

R: 5'-AGTGGTATAGACAGGTCTGTTGG-3'

F: $5^{\prime}$-TGGTTTGCCATCGTTTTGCTG-3'

R: 5'-ACAGGTGAGGTTCACTGTTTCT-3'

F: $5^{\prime}$-CAGGCGGTGCCTATGTCTC-3'

R: 5'-CGATCACCCCGAAGTTCAGTAG-3'

F: $5^{\prime}$-TATCATCACCTTCCCGCTG-3'

R: 5'-GTCATATGTTACCAGCTCTG-3'

F: 5'-AGCCCTCGCCCACAACTTGC-3'

R: $5^{\prime}$-TGACCCCCGGCTTCCGTTCA-3'

(Abcam, ab54481), RGC32, and $\alpha$-tubulin (Cell Signaling Technology, 9099S) were used. All the antibodies were applied at 1:1000 dilution. Protein expressions were detected using an enhanced chemiluminescence kit (Millipore).

\section{Histological analysis}

After fixing in $4 \%$ paraformaldehyde overnight, the epididymal fat was dehydrated and embedded in paraffin. The sections $(5 \mu \mathrm{m})$ were cut with a microtome. The sections were deparaffinized and stained with hematoxylin and eosin (H\&E). Images were captured by a Nikon microscope. For quantitative analysis of adipocyte area, eight images of H\&E-stained sections were acquired from each animal, and the cross-sectional area of each adipocyte was measured using ImageJ software.

\section{Statistical analyses}

Data are presented as means \pm s.D., and the numbers of independent experiments are indicated for each data set. For statistical analysis, the two groups were compared using two-tailed Student's $t$-tests, and the four groups were

Published by Bioscientifica Ltd. 
evaluated by two-way ANOVA followed by Bonferroni post hoc tests for multiple comparisons, whereas the three and five groups were evaluated by one-way ANOVA followed by Tukey's multiple comparisons using GraphPad Prism 5.0 software (La Jolla, CA, USA). $P<0.05$ was considered statistically significant.

\section{Results}

\section{RGC32 deficiency prevented HFD-induced obesity}

Our previous study has shown that the body size of $\mathrm{RgC}_{3} 2^{-/-}$mice is smaller compared with their WT littermates when they are born (Cui et al. 2013). Interestingly, the difference is not as dramatic as they age, suggesting that RGC32 has little effect on the postnatal growth on the regular chow diet. It is unknown, however, whether RGC32 affects HFD-induced obesity. To test this, we fed WT mice with HFD for 12 weeks and then detected RGC32 expression in adipose tissue. We found that RGC32 expression was dramatically upregulated by the HFD (Fig. 1A). To investigate the potential role of RGC32 in obesity, the WT and $R g c 32^{-/-}$mice were fed with HFD for 12 weeks. The HFD-fed WT mice gained significantly more weight than the normal chow controls. However, $R g c 32^{-/-}$appeared to diminish the weight gain (Fig. 1B). The weight of epididymal fat pads was also markedly lower in HFD-fed $R g c 32^{-/-}$mice as compared to HFD-fed WT mice, although it was increased compared with the normal chow controls (Fig. 1C). Histological analysis of epididymal fat showed that HFD induced a significant adipocyte hypertrophy (more than fivefold) in WT mice. However, this effect was significantly reduced in $R g c 32^{-/-}$mice (Fig. 1D and E). To determine whether the lean phenotype of $\mathrm{Rgc}_{\mathrm{c}} 2^{-/-}$mice was due to a reduced energy intake, we housed the mice individually in metabolic cages and monitored the food intake. As shown in Fig. 1F, the energy intake of WT and $R g c 32^{-/-}$mice fed with HFD was increased compared with the normal chow controls, while there was no difference between WT and $\mathrm{RgC}_{32} 2^{-/}$mice fed on either normal chow or HFD. There were also no significant differences in the water intake, urine, and feces (data not shown). To assess the energy expenditure, we measured the body weight before and after an 8-h fast. In the absence of energy intake, greater loss of body weight indicates increased energy expenditure. After fasting, although there was no significant difference between WT and $\mathrm{Rgc}_{\mathrm{C}} \mathrm{2}^{-/-}$mice under chow conditions,

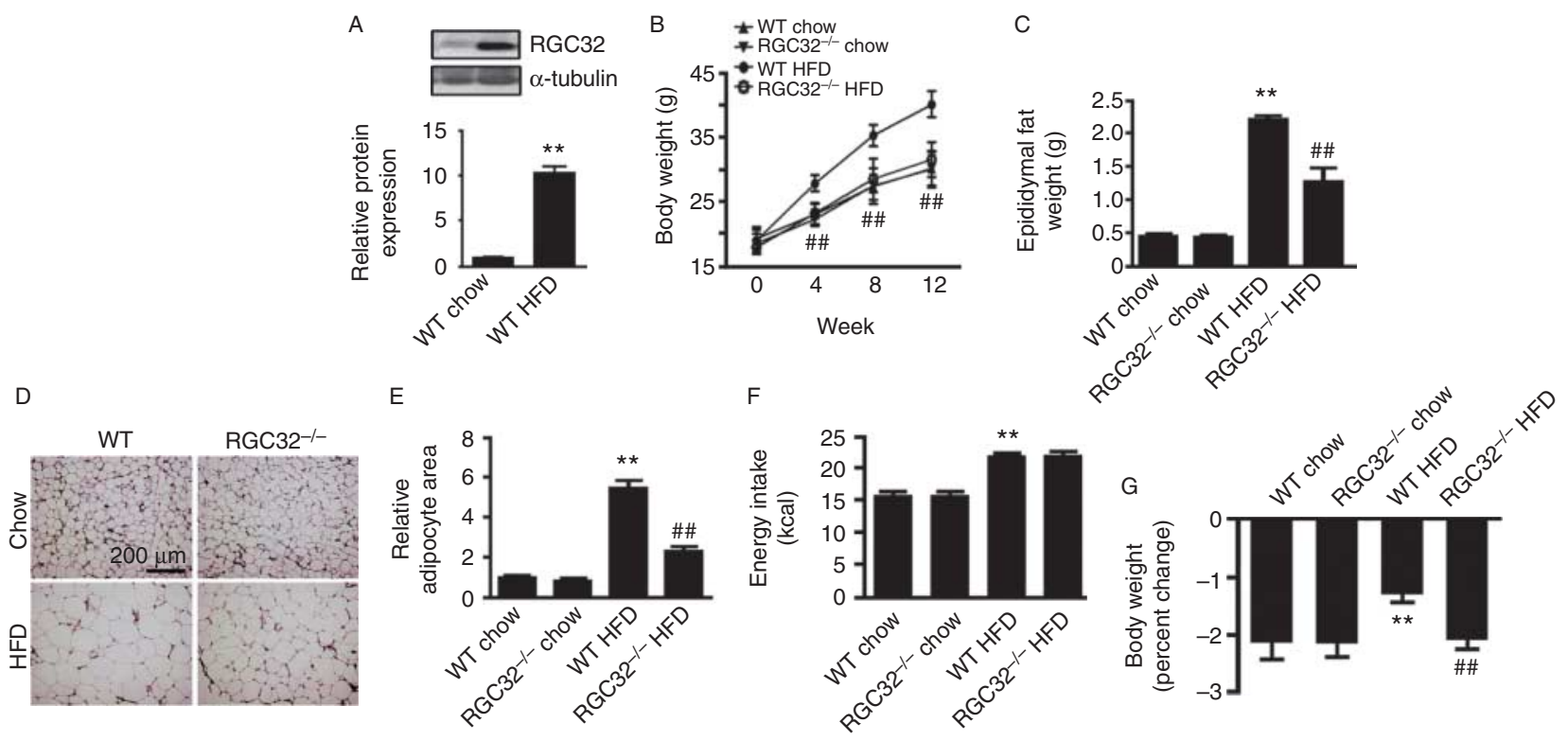

Figure 1

RGC32 deficiency prevented HFD-induced obesity. (A) RGC32 expression in adipose tissue of WT mice fed with normal chow or a 12-week high-fat diet (HFD) were detected by western blotting and normalized to $\alpha$-tubulin $(n=3)$. (B) Body weight of WT and $R g c 32^{-1-}$ mice fed with normal chow $(n=6)$ or HFD $(n=10)$. (C) Weights of epididymal fat from WT and $R g c 32^{-1-}$ mice fed on normal chow and HFD. (D) Representative H\&E-stained images of epididymal fat from WT and $R g c 32^{-1-}$ mice fed on normal chow http://joe.endocrinology-journals.org DOI: $10.1530 / J O E-14-0548$
() 2015 Society for Endocrinology Printed in Great Britain and HFD. (E) Quantitative analysis of the mean adipocyte area. The areas were normalized to the mean adipocyte area of WT mice fed on normal chow. (F) Energy intake of WT and $R g c 32^{-1-}$ mice fed on normal chow and HFD. (G) Body weight change of WT and $R g c 32^{-1-}$ mice after an 8-h fast. ${ }^{*} P<0.01$ compared with WT chow group, ${ }^{\# \#} P<0.01$ compared with WT HFD group. A full colour version of this figure is available at http://dx.doi. org/10.1530/JOE-14-0548. 
HFD-fed $\mathrm{Rgc} 32^{-1-}$ mice lost more body weight than HFDfed WT mice (Fig. 1G), suggesting that the energy expenditure was increased in HFD-fed $\mathrm{Rgc}_{\mathrm{C}} \mathrm{2}^{-/-}$mice, which may, at least partially, be responsible for the lean phenotype of HFD-fed $R g c 32^{-/-}$mice.

\section{RGC32 deficiency improved metabolic homeostasis in HFD-fed mice}

Diet-induced obesity is typically accompanied by dyslipidemia and insulin resistance. Therefore, we measured serum triglyceride and cholesterol concentrations. No difference was observed between WT and $\mathrm{Rgc}_{3} 2^{-/-}$mice on normal chow (Fig. 2A and B). However, on HFD, WT mice exhibited significantly increased serum concentrations of triglyceride, HDL cholesterol, and LDL/VLDL cholesterol (Fig. 2A and B). Importantly, $R g c 32^{-/-}$mice appeared to be resistant to the HFD-induced increase in serum triglyceride and cholesterol. The serum triglyceride and LDL/VLDL cholesterol concentrations in $\mathrm{Rgc}_{3} 2^{-/-}$ mice were not altered by the HFD feeding and thus were much lower compared with the HFD-fed WT control. HDL cholesterol was slightly lower in HFD-fed $\mathrm{Rgc}_{3} 2^{-1-}$ mice than the WT control, although it was increased compared with $R g c 32^{-1-}$ mice fed with normal chow (Fig. 2A and B).

To determine whether RGC32 affects insulin sensitivity, blood glucose and serum insulin levels were detected. $R g c 32^{-/-}$mice showed similar fasting blood glucose and insulin levels compared with WT mice fed with normal chow (Fig. 2C and D). Thus, the homeostasis model assessment-insulin resistance (HOMA-IR) scores had no difference (Fig. 2E). HFD significantly increased fasting blood glucose level, insulin concentration, and HOMA-IR score in WT mice but not in $\mathrm{Rgc}_{3} 2^{-1-}$ mice (Fig. 2C, D, and E), suggesting that insulin sensitivity is improved due to RGC32 deficiency. Improved insulin sensitivity in HFD-fed $R g c 32^{-/-}$mice was also confirmed using ITT, although there was no difference between WT and $\mathrm{RgC} 2^{-/-}$mice fed with normal chow (Fig. 3A, B, and C). Furthermore, GTT showed that on normal chow, blood glucose levels appeared to be improved at 15 and 30 min (Fig. 3D), and the area under the curve (AUC) was significantly lower in $\mathrm{Rgc}_{3} 2^{-/-}$mice compared with WT controls (Fig. 3F), indicating that the glucose tolerance was improved in $\mathrm{Rgc}_{3} 2^{-/-}$mice under chow conditions. HFD-fed WT mice developed severe glucose intolerance,
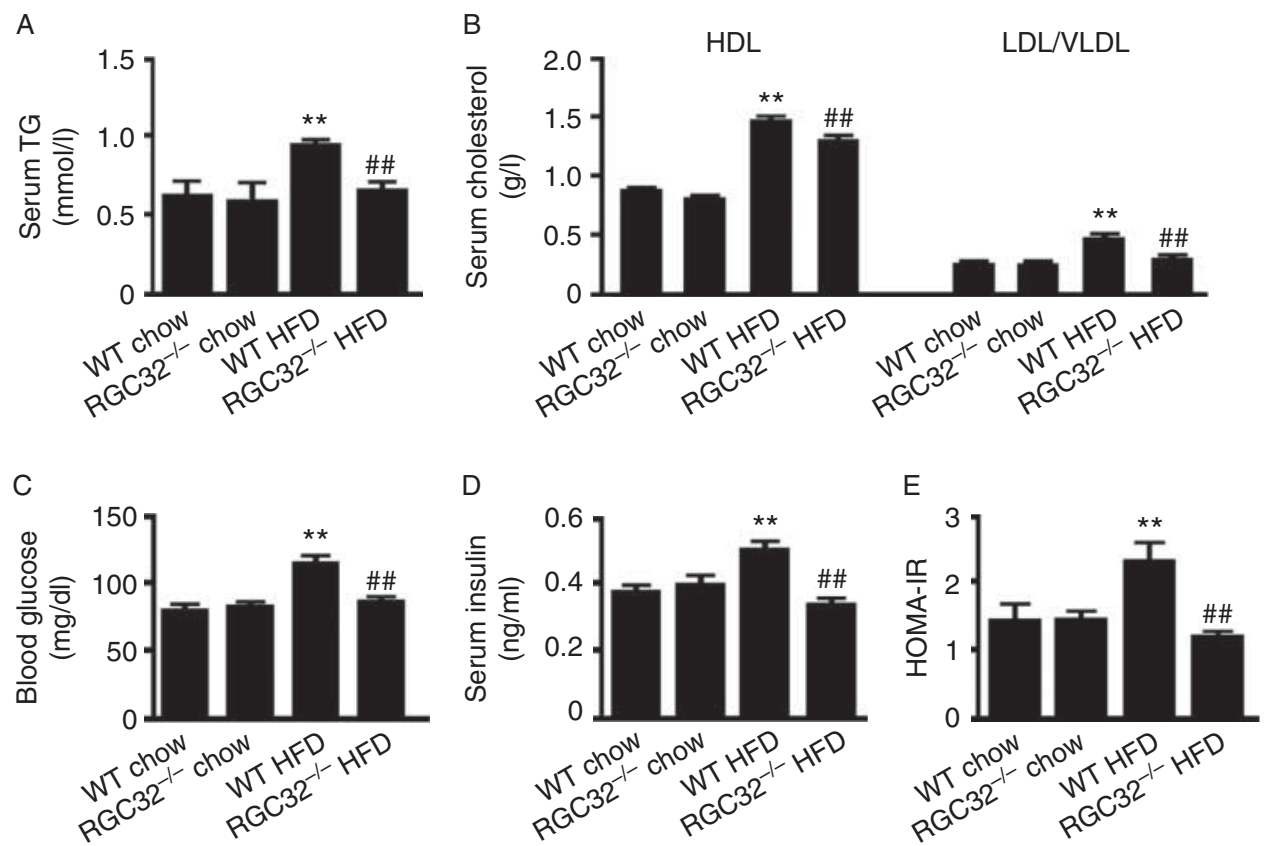

\section{Figure 2}

RGC32 deficiency improved metabolic homeostasis in HFD-fed mice. (A) Serum triglyceride (TG), (B) HDL cholesterol, and LDL/VLDL cholesterol concentrations in WT and $R g c 32^{-1-}$ mice fed on normal chow and HFD $(n=6)$. (C) Fasting blood glucose, (D) insulin concentration, and
(E) homeostasis model assessment-insulin resistance (HOMA-IR = fasting glucose $\times$ fasting insulin/22.5) in WT and $R g c 32^{-1-}$ mice fed on normal chow and HFD $(n=6)$. ${ }^{*} P<0.01$ compared with WT chow group, ${ }^{\# \#} P<0.01$ compared with WT HFD group. 

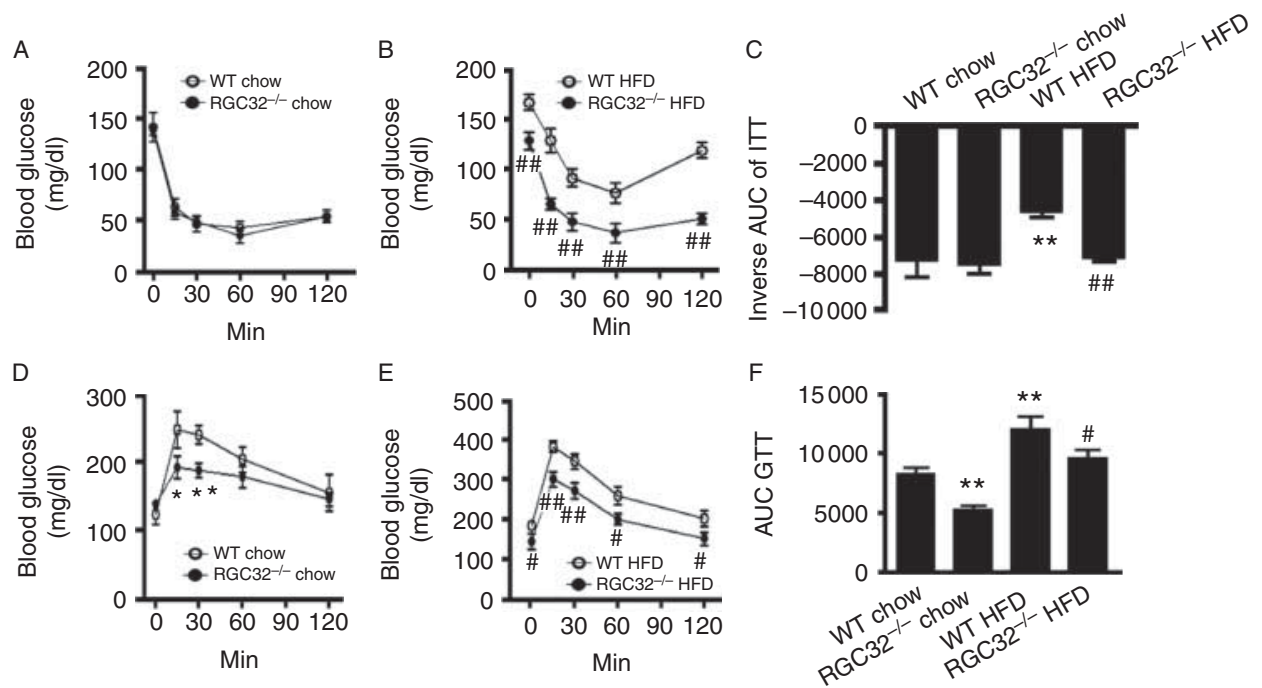

Figure 3

RGC32 deficiency prevents HFD-induced insulin resistance in mice. (A and B) Insulin tolerance test (ITT) in WT and $R g c 32^{-1-}$ mice fed on normal chow (A) and HFD (B) $(n=6)$. (C) Inverse area under the curve (AUC) of ITT. (D and E) Glucose tolerance test (GTT) in WT and $R g c 32^{-1-}$ mice fed on

whereas $R g c 32^{-/-}$mice showed a significantly improved glucose tolerance compared with the HFD-fed WT mice (Fig. 3E and F). These data demonstrate that RGC32 deficiency improves glucose tolerance and protects mice from HFD-induced insulin resistance.

\section{RGC32 deficiency attenuated adipose tissue and systemic inflammation in HFD-fed mice}

Since adipose tissue inflammation and low levels of systemic inflammation are important factors contributing to the development of obesity, we detected adipose tissue and serum inflammatory status of the mice. RGC32 deficiency appeared to decrease Il 6 and Tnf $\alpha$ mRNA expression in the fat tissue under chow conditions, while had no effect on other adipokines such as adiponectin, leptin, and IL12 (Fig. 4A and B). HFD dramatically decreased adiponectin, an anti-inflammatory adipokine, while increased the expression of pro-inflammatory adipokines leptin, IL6, TNF $\alpha$, and IL12 in the fat tissue of WT mice. However, these effects were alleviated in $R g c 32^{-/-}$mice (Fig. 4A and B). Similar results were observed with the circulating adipokines (Fig. 4C and D), although the circulating IL6, TNF $\alpha$, and IL12 were undetectable under chow conditions (data not shown). These data demonstrate that RGC32 deficiency attenuates adipose tissue and systemic inflammation in HFD-fed mice. normal chow (D) and HFD (E) ( $n=6)$. (F) Quantification of the AUC of GTT. ${ }^{*} P<0.05,{ }^{* *} P<0.01$ compared with WT chow group, ${ }^{\#} P<0.05,{ }^{\# \#} P<0.01$ compared with WT HFD group.

\section{RGC32 deficiency increased the expression of metabolic genes in adipose tissues}

Since HFD-fed $\mathrm{Rgc}_{3} 2^{-/-}$mice had reduced fat mass and improved adipose tissue inflammation, we further assessed the expression of metabolic genes related to $\beta$-oxidation, lipolysis, and thermogenesis in adipose tissue. As shown in Fig. 5A, RGC32 deficiency increased the mRNA expression of Ppara, Lipe, and Pgc1 $\alpha$ in epididymal fat tissue as compared with WT controls both under normal chow and HFD conditions. Since the alterations in HFD groups were greater than those in the normal chow groups, and the body weight difference was observed in HFD groups, we confirmed the protein expression of these genes in the epididymal adipose tissue of HFD-fed mice (Fig. 5B and C).

It is known that PGC1 $\alpha$ can induce browning of subcutaneous adipose tissue through regulation of uncoupling protein 1 (UCP1) (Bostrom et al. 2012). We further determined adipose tissue browning in interscapular and inguinal fat tissues of WT and $R g c 32^{-/-}$mice. As shown in Fig. 5D and E, PGC1 $\alpha$, UCP1, and PRDM16 were all increased in both interscapular and inguinal fat tissues of Rgc $32^{-/-}$mice compared with WT controls under both normal chow and HFD conditions. These data suggest that RGC32 deficiency induces browning of adipose tissues, leading to increased energy expenditure. 


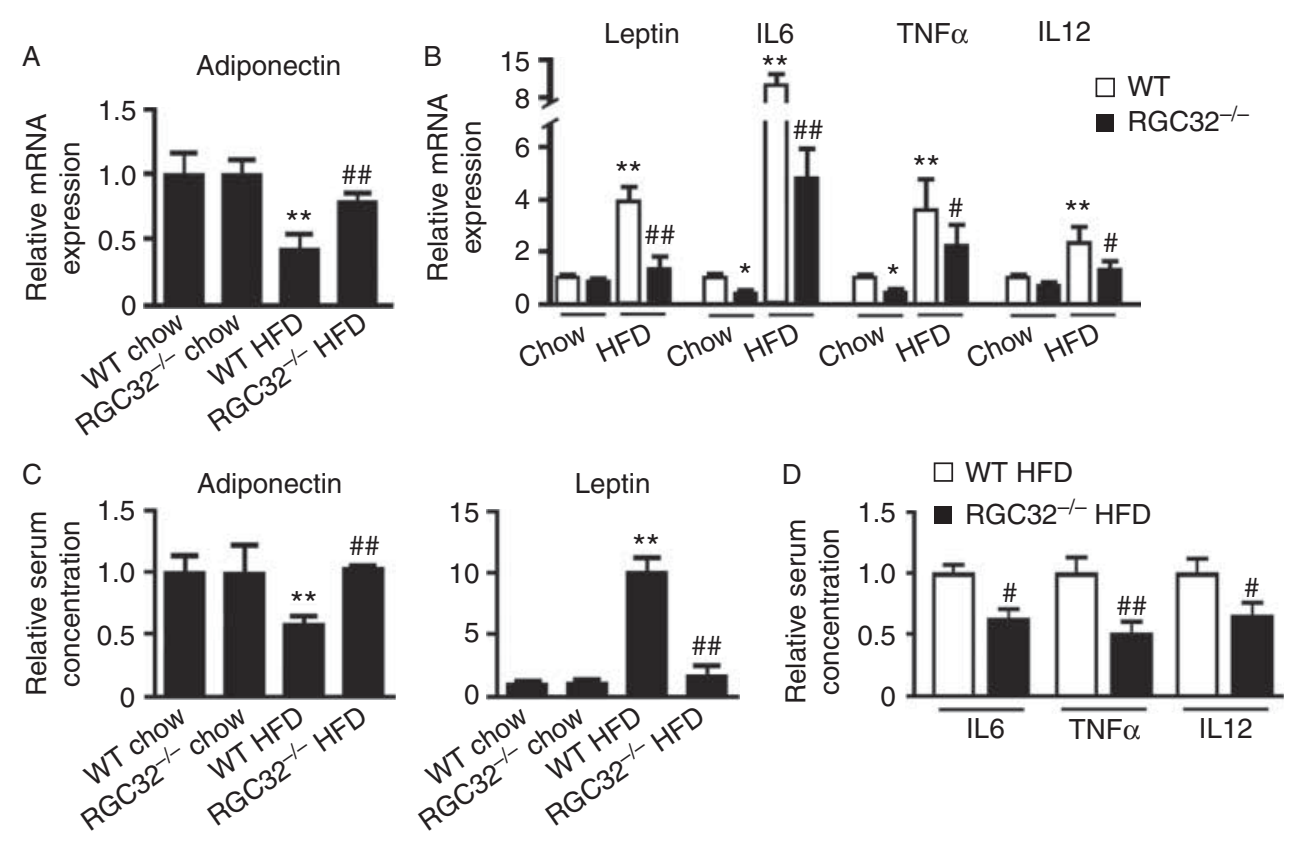

\section{Figure 4}

RGC32 deficiency attenuated adipose tissue and systemic inflammation in HFD-fed mice. (A and B) mRNA expression of adiponectin, leptin, interleukin 6 (IL6), tumor necrosis factor $\alpha$ (TNF $\alpha$ ), and IL12 in epididymal adipose tissue from WT and $R g c 32^{-1-}$ mice $(n=6)$ was measured by qPCR.

\section{RGC32 had no effect on adipocyte differentiation}

Since HFD induced a significant adipocyte hypertrophy in WT mice (Fig. 1D and E), we sought to determine whether RGC32 promotes adipocyte differentiation. RGC32 has been shown to induce smooth muscle cell differentiation (Li et al. 2007). In the differentiation of 3T3-L1 preadipocytes, RGC32 expression was dramatically increased accompanied with elevated expression of adipogenic differentiation specific genes Ppary and C/Ebp $\alpha$ (Fig. 6A, B, and C). To test RGC32 function in this process, we knocked down or overexpressed RGC32 in 3T3-L1 cells followed by induction of adipocyte differentiation and found that neither knockdown nor overexpression of RGC32 had any effect on the expression of adipogenic-specific genes (Fig. 6D) or lipid accumulation (Fig. 6E and F). These data indicate that the role of RGC32 in obesity was not due to an increased adipocyte differentiation. To confirm the regulation of energy expenditure-related genes by RGC32 as observed in the fat tissue (shown in Fig. 5A, B, and C), we detected the protein expression of PPAR $\alpha$, LIPE, and PGC1 $\alpha$ in adipocytes differentiated from 3T3-L1 cells. Consistently, all of these genes were increased when RGC32 was knocked down (Fig. 6G and H). The expression of Lipe
(C and D) Protein concentration of adiponectin, leptin, IL6, TNF $\alpha$, and IL12 in the serum from WT and $R g c 32^{-1-}$ mice $(n=6)$ was measured by cytometric bead array immunoassay. ${ }^{*} P<0.05, * * P<0.01$ compared with WT chow group, ${ }^{\#} P<0.05,{ }^{\# \#} P<0.01$ compared with WT HFD group.

and $P g c 1 \alpha$ was decreased when RGC32 was overexpressed. Ppar $\alpha$ expression appeared not to be dramatically affected by RGC32 overexpression (Fig. 6G and H). These data indicate that RGC32 deficiency prevents mice from developing diet-induced obesity through the upregulation of energy expenditure-related genes in adipose tissues.

\section{Discussion}

Our present study demonstrates for the first time that RGC32 plays an important role in HFD-induced obesity. RGC32 is strongly upregulated in adipose tissue of HFD-fed mice. RGC32 deficiency prevents the development of HFD-induced obesity and insulin resistance because $R g c 32^{-/-}$mice fed a HFD gain less weight without change in energy intake and have a reduced fat mass. Interestingly, HFD increases the fat mass of $R g c 32^{-/-}$mice without altering their body weight, which is probably because the increase in the fat mass in these mice accounts for a relatively small portion of the whole body mass and thus does not cause a significant change in their body weight (as shown in Fig. 1B). HFD-fed $R g c 32^{-/-}$mice also exhibit significantly improved insulin sensitivity as indicated by improved HOMA-IR and insulin tolerance. Our results are consistent with previous studies showing that HFD causes

Published by Bioscientifica Ltd. 

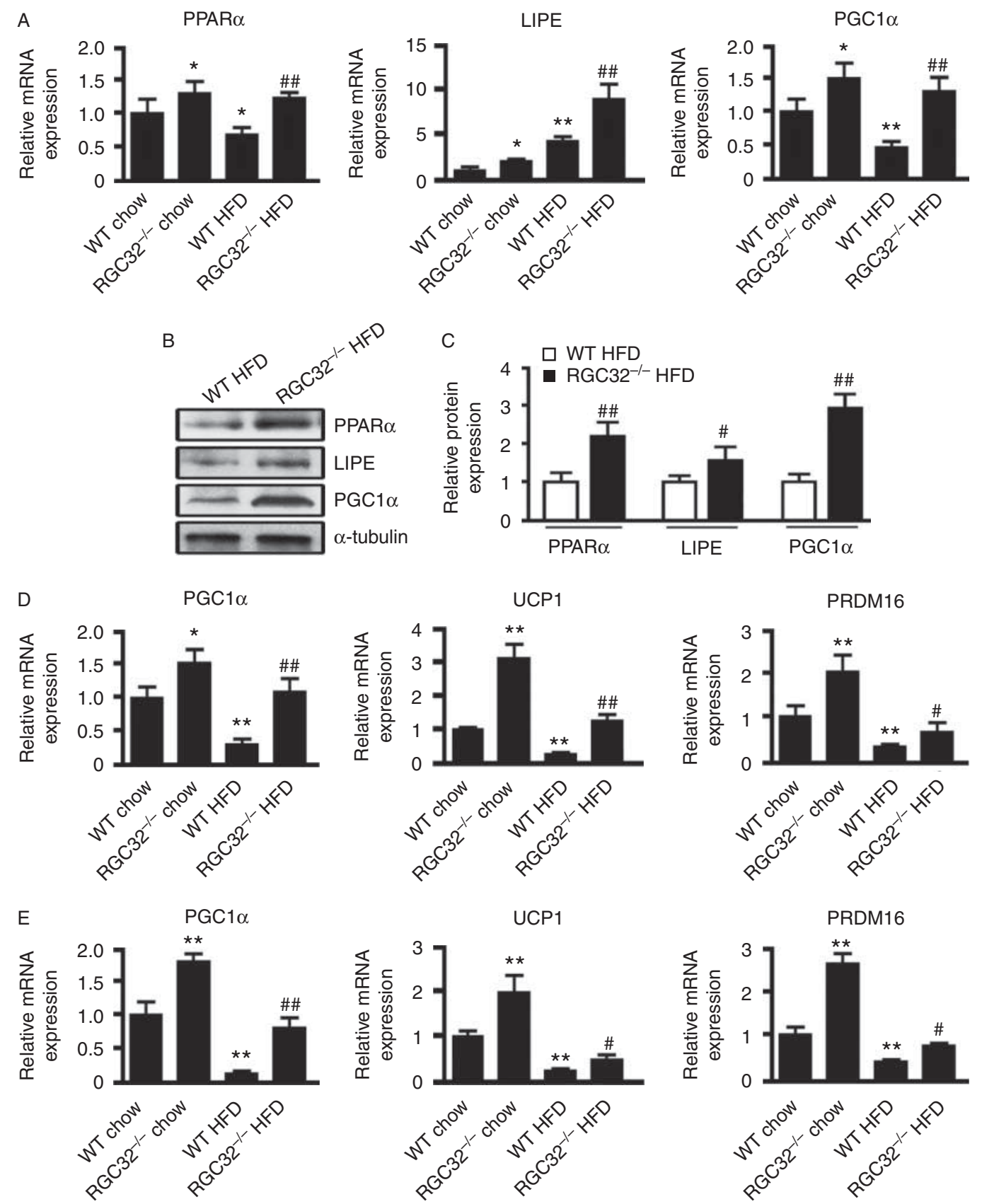

Figure 5

RGC32 deficiency increased the expression of metabolic genes in adipose tissues. (A) mRNA expression of Ppar $\alpha$, Lipe, and Pgc1 $\alpha$ in the epididymal adipose tissues of WT and $R g c 32^{-1-}$ mice $(n=6)$. (B and C) PPAR $\alpha$, LIPE, and PGC1 $\alpha$ protein expression in the epididymal adipose tissues of WT and $R g C 32^{-1-}$ mice were detected by western blotting analysis and normalized

obesity and insulin resistance (Winzell \& Ahren 2004), which is a characteristics of type 2 diabetes and several cardiometabolic syndromes including hypertension and dyslipidemia (Kim et al. 2008). Although adipocyte differentiation is involved in diet-induced obesity (Berry to $\alpha$-tubulin ( $n=6$ ). (D and E) mRNA expression of Pgc1 $\alpha, U c p 1$, and Prdm 16 in the interscapular (D) and inguinal (E) fat tissues of WT and Rgc32 $^{-/-}$ mice $(n=6)$. ${ }^{*} P<0.05, * * P<0.01$ compared with WT chow group, ${ }^{\#} P<0.05,{ }^{\# \#} P<0.01$ compared with WT HFD group.

et al. 2012, Federico et al. 2012), it may not be involved in RGC32-mediated obesity because either overexpression or knockdown of RGC32 has no effect on the expression of adipogenic differentiation-specific genes Ppar $\gamma$ and C/Ebp $\alpha$ and lipid accumulation of the adipocytes. 

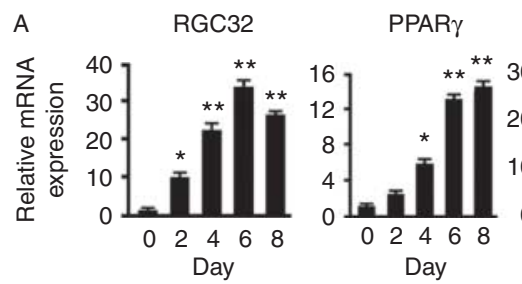

$\mathrm{C} / \mathrm{EBP} \alpha$

B
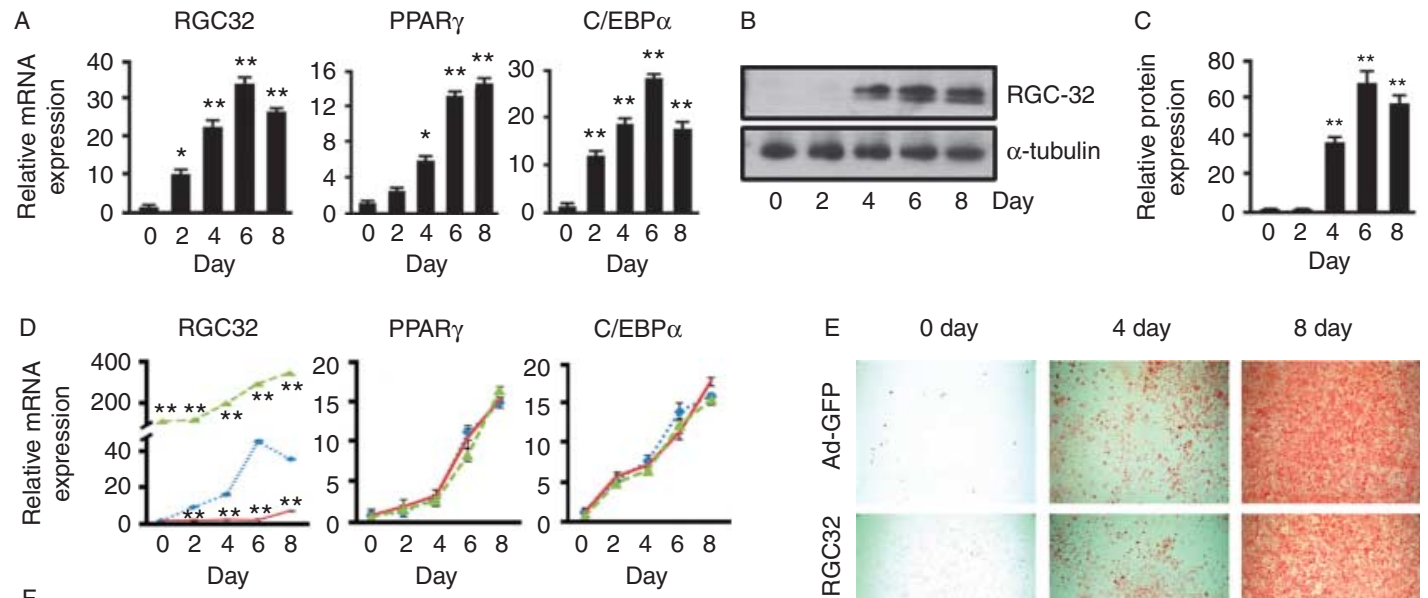

PPAR $\gamma$
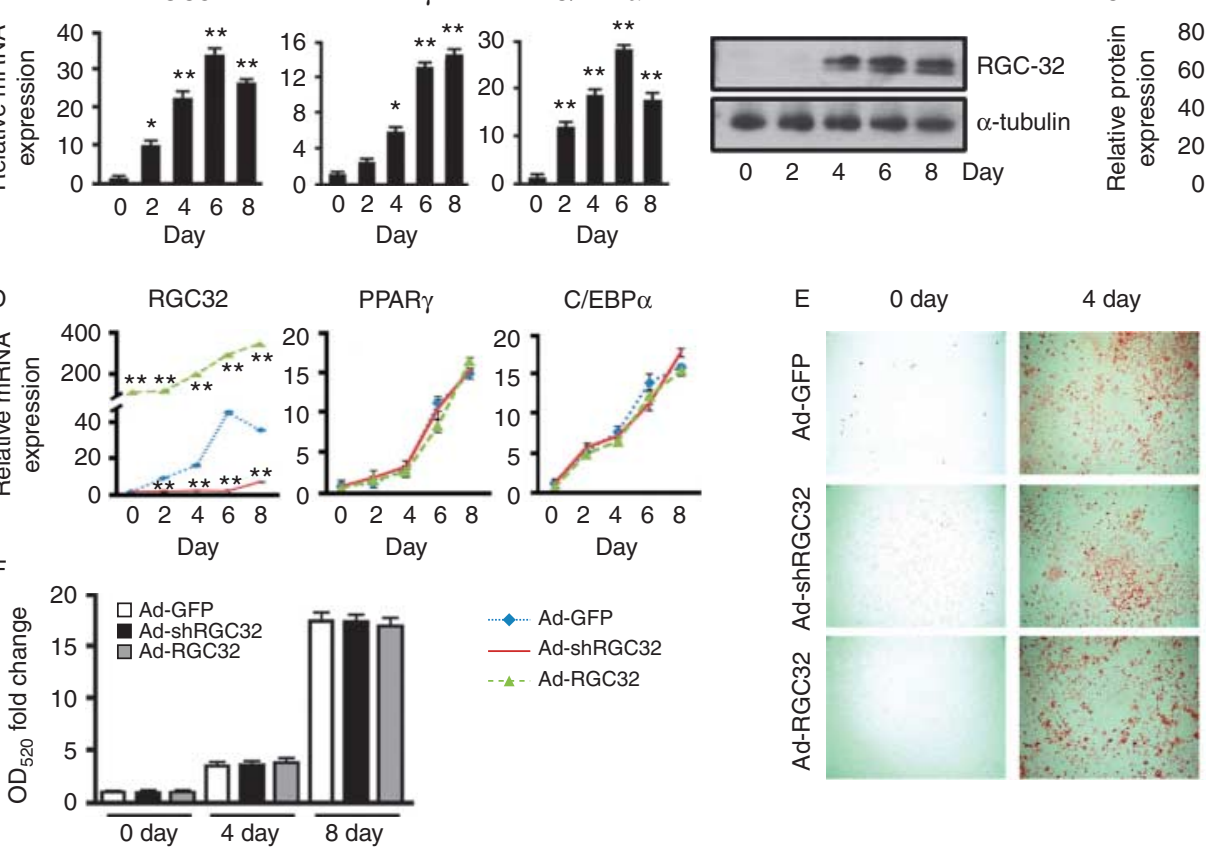

8 day

G

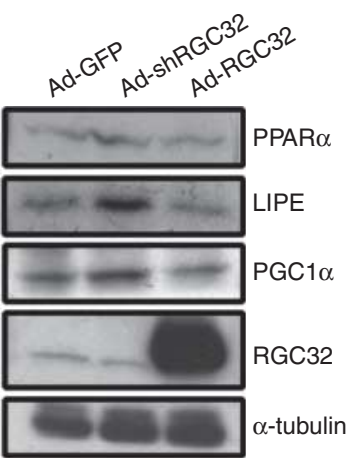

...... Ad-GFP

Ad-shRGC32

-1-Ad-RGC32

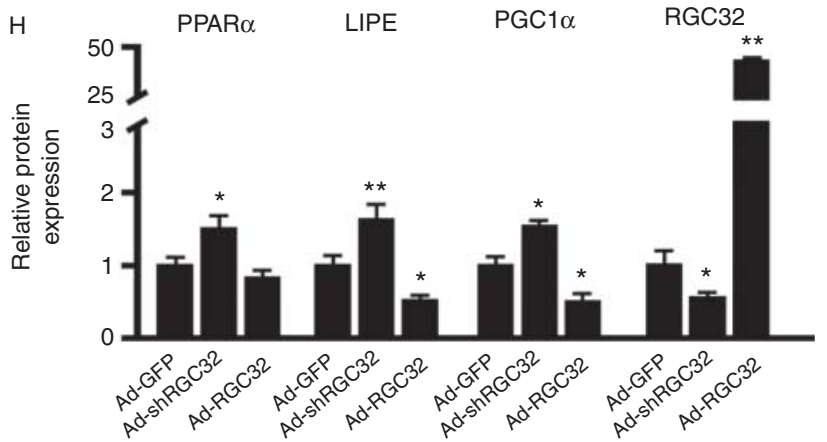

\section{Figure 6}

RGC32 had no effect on adipocyte differentiation. (A) mRNA expression of $R g c 32$, Ppary, and C/Ebp $\alpha$ during 3T3-L1 preadipocyte differentiation at the indicated times. (B and C) RGC32 protein expression during 3T3-L1 preadipocyte differentiation was detected by western blotting analysis and normalized to $\alpha$-tubulin. ${ }^{*} P<0.05$, $* * P<0.01$ compared with vehicletreated group ( 0 day). ( $D, E, F, G$, and $H$ ) 3T3-L1 preadipocyte was transduced with Ad-GFP, Ad-shRGC32, or Ad-RGC32 for $24 \mathrm{~h}$ and then was induced for adipocyte differentiation. (D) mRNA expression of Rgc32,

The protection from diet-induced obesity in $\mathrm{Rgc}_{\mathrm{c}} 32^{-/-}$ mice is linked to the attenuated adipose tissue and systemic inflammation and increased adipose tissue energy expenditure. Recent data have shown that adipose tissue inflammation is a key mechanism leading to obesity and insulin resistance (Chakrabarti et al. 2009, Lee et al. 2010). RGC32 is essential for C5b-9-induced cell cycle activation (Fosbrink et al. 2009), indicating an important role in the regulation of inflammation. Indeed, RGC32 deficiency suppresses adipose tissue and systemic
Ppary, and C/Ebp $\alpha$ and (E and F) lipid droplet accumulation was determined at the indicated times. (G and H) PPAR $\alpha$, LIPE, PGC $1 \alpha$, and RGC32 expression was detected by western blotting analysis and normalized to $\alpha$-tubulin. ${ }^{*} P<0.05,{ }^{*} P<0.01$ compared with Ad-GFP group. All results are representatives of at least three independent experiments. A full colour version of this figure is available at http://dx.doi. org/10.1530/JOE-14-0548.

inflammation in HFD-fed mice, as evidenced by the reduced production of proinflammatory adipokines including leptin, IL6, TNF $\alpha$, and IL12 and increased antiinflammatory adipokine adiponectin. Dietary excess and obesity have been shown to cause lipid accumulation in adipocytes, initiating a state of cellular stress and activation of NFкB signaling pathway (Shoelson et al. 2006), leading to an increased adipocyte production of proinflammatory cytokines. Our previous study has shown that RGC32 activates NFאB in vascular endothelial 
cells (Cui et al. 2013). Therefore, RGC32 may increase inflammation of adipose tissue through activating NF-кB signaling pathway, which may be studied in the future.

RGC32 appears to regulate obesity development by influencing the thermogenesis. Particularly, RGC32 may suppress the expression of metabolic genes PPAR $\alpha$, Lipe, and PGC1 $\alpha$ because dramatic elevation of PPAR $\alpha$, LIPE, and PGC1 $\alpha$ is observed in the adipose tissue of HFD-fed $\mathrm{RgC} 32^{-/-}$mice. LIPE is a rate-limiting enzyme to cleave fatty acids from the triglyceride molecule (Schweiger et al. 2006). PPAR $\alpha$ promotes uptake, utilization, and catabolism of fatty acids by upregulating the genes involved in fatty acid transport; fatty acid binding and activation; and peroxisomal and mitochondrial fatty acid $\beta$-oxidation (Rakhshandehroo et al. 2010). PGC1 $\alpha$ enhances thermogenesis and oxidative metabolism of adipose tissue (Liang \& Ward 2006, Jun et al. 2014). Indeed, RGC32 deficiency promotes the browning of adipose tissues. Brown adipose tissue is known to dissipate chemical energy and protect against obesity through a process termed as nonshivering thermogenesis (Bi et al. 2014). Active brown adipose tissue burns lipids to produce heat, resulting in an increase in energy expenditure. $P G C 1 \alpha$ and $U C P 1$ are highly expressed in brown adipose tissue (Fisher et al. 2012), and PRDM16 is a brown adipose determination factor (Seale et al. 2011). Increased expression of these genes in interscapular and inguinal fat tissues may have collectively caused the increased energy expenditure and the lean phenotype of HFD-fed $\mathrm{RgC}_{\mathrm{g}} 2^{-/-}$mice. Besides adipose tissue, defective energy expenditure of skeletal muscle also contributes to diet-induced obesity and insulin resistance. However, since RGC32 expression is undetectable in skeletal muscle, contribution of skeletal muscle energy expenditure to the lean phenotype of HFD-fed $R g c 32^{-/-}$mice is likely to be minimal.

In summary, our study demonstrates that RGC32 mediates HFD-induced obesity through enhancing inflammation while decreasing energy expenditure in adipose tissues.

\section{Declaration of interest}

The authors declare that there is no conflict of interest that could be perceived as prejudicing the impartiality of the research reported.

\section{Funding}

This work was supported by NIH grants HL107526, HL119053, and HL123302 and National Natural Science Foundation of China grant 81328002 (to S-Y C). X-B C is supported by an American Heart Association Postdoctoral Fellowship (14POST20480015).

\section{Author contribution statement}

$\mathrm{X}-\mathrm{B} \mathrm{C}$ conceived and carried out the experiments including data collection, analysis and interpretation, and wrote the manuscript. J-N L performed the $\mathrm{GPCR}$, western blotting analysis and adipocyte differentiation experiments. J $Y$ offered critical logistical advice on experimental design and data interpretation and critically read the manuscript. S-Y C is the guarantor of this work and, as such, had full access to all the data in the study and takes responsibility for the integrity of the data and the accuracy of the data analysis. All authors had final approval of the submitted and published versions.

\section{References}

Badea TC, Niculescu FI, Soane L, Shin ML \& Rus H 1998 Molecular cloning and characterization of RGC-32, a novel gene induced by complement activation in oligodendrocytes. Journal of Biological Chemistry 273 26977-26981. (doi:10.1074/jbc.273.41.26977)

Bandyopadhyay P 2009 The role of incretin-based therapies in the management of type 2 diabetes. Drug News \& Perspectives 22 559-567. (doi:10.1358/dnp.2009.22.9.1437963)

Berry DC, DeSantis D, Soltanian H, Croniger CM \& Noy N 2012 Retinoic acid upregulates preadipocyte genes to block adipogenesis and suppress diet-induced obesity. Diabetes 61 1112-1121. (doi:10.2337/db11-1620)

Bi P, Shan T, Liu W, Yue F, Yang X, Liang XR, Wang J, Li J, Carlesso N, Liu X et al. 2014 Inhibition of Notch signaling promotes browning of white adipose tissue and ameliorates obesity. Nature Medicine 20 911-918. (doi:10.1038/nm.3615)

Bostrom P, Wu J, Jedrychowski MP, Korde A, Ye L, Lo JC, Rasbach KA, Bostrom EA, Choi JH, Long JZ et al. 2012 A PGC1- $\alpha$-dependent myokine that drives brown-fat-like development of white fat and thermogenesis. Nature 481 463-468. (doi:10.1038/nature10777)

Chakrabarti SK, Cole BK, Wen Y, Keller SR \& Nadler JL 2009 12/15lipoxygenase products induce inflammation and impair insulin signaling in 3T3-L1 adipocytes. Obesity 17 1657-1663. (doi:10.1038/oby.2009.192)

Chatterjee TK, Basford JE, Knoll E, Tong WS, Blanco V, Blomkalns AL, Rudich S, Lentsch AB, Hui DY \& Weintraub NL 2014 HDAC9 knockout mice are protected from adipose tissue dysfunction and systemic metabolic disease during high-fat feeding. Diabetes 63 176-187. (doi:10.2337/db13-1148)

Cui XB, Wang C, Li L, Fan D, Zhou Y, Wu D, Cui QH, Fu FY \& Wu LL 2012 Insulin decreases myocardial adiponectin receptor 1 expression via PI3K/Akt and FoxO1 pathway. Cardiovascular Research 93 69-78. (doi:10.1093/cvr/cvr273)

Cui XB, Guo X \& Chen SY 2013 Response gene to complement 32 deficiency causes impaired placental angiogenesis in mice. Cardiovascular Research 99 632-639. (doi:10.1093/cvr/cvt121)

Distefano JK \& Watanabe RM 2010 Pharmacogenetics of anti-diabetes drugs. Pharmaceuticals 3 2610-2646. (doi:10.3390/ph3082610)

Federico L, Ren H, Mueller PA, Wu T, Liu S, Popovic J, Blalock EM, Sunkara M, Ovaa H, Albers HM et al. 2012 Autotaxin and its product lysophosphatidic acid suppress brown adipose differentiation and promote diet-induced obesity in mice. Molecular Endocrinology 26 786-797. (doi:10.1210/me.2011-1229)

Fisher FM, Kleiner S, Douris N, Fox EC, Mepani RJ, Verdeguer F, Wu J, Kharitonenkov A, Flier JS, Maratos-Flier E et al. 2012 FGF21 regulates PGC- $1 \alpha$ and browning of white adipose tissues in adaptive thermogenesis. Genes and Development 26 271-281. (doi:10.1101/ gad.177857.111)

Fosbrink M, Cudrici C, Niculescu F, Badea TC, David S, Shamsuddin A, Shin ML \& Rus H 2005 Overexpression of RGC-32 in colon cancer and other tumors. Experimental and Molecular Pathology 78 116-122. (doi:10.1016/j.yexmp.2004.11.001)

Published by Bioscientifica Ltd. 
Fosbrink M, Cudrici C, Tegla CA, Soloviova K, Ito T, Vlaicu S, Rus V, Niculescu F \& Rus H 2009 Response gene to complement 32 is required for C5b-9 induced cell cycle activation in endothelial cells. Experimental and Molecular Pathology 86 87-94. (doi:10.1016/j.yexmp.2008.12.005)

Friedman JM 2003 A war on obesity, not the obese. Science 299 856-858. (doi:10.1126/science.1079856)

Hotamisligil GS 2006 Inflammation and metabolic disorders. Nature $\mathbf{4 4 4}$ 860-867. (doi:10.1038/nature05485)

Jun HJ, Joshi Y, Patil Y, Noland RC \& Chang JS 2014 NT-PGC-1 $\alpha$ activation attenuates high-fat diet-induced obesity by enhancing brown fat thermogenesis and adipose tissue oxidative metabolism. Diabetes 63 3615-3625. (doi:10.2337/db13-1837)

Kanda H, Tateya S, Tamori Y, Kotani K, Hiasa K, Kitazawa R, Kitazawa S, Miyachi H, Maeda S, Egashira K et al. 2006 MCP-1 contributes to macrophage infiltration into adipose tissue, insulin resistance, and hepatic steatosis in obesity. Journal of Clinical Investigation 116 1494-1505. (doi:10.1172/JCI26498)

Kim JA, Wei Y \& Sowers JR 2008 Role of mitochondrial dysfunction in insulin resistance. Circulation Research 102 401-414. (doi:10.1161/ CIRCRESAHA.107.165472)

Kim DS, Lee JY, Lee SM, Choi JE, Cho S \& Park JY 2011 Promoter methylation of the RGC32 gene in nonsmall cell lung cancer. Cancer 117 590-596. (doi:10.1002/cncr.25451)

Lee SJ, Kim JY, Nogueiras R, Linares JF, Perez-Tilve D, Jung DY, Ko HJ, Hofmann SM, Drew A, Leitges M et al. 2010 PKCzeta-regulated inflammation in the nonhematopoietic compartment is critical for obesity-induced glucose intolerance. Cell Metabolism 12 65-77. (doi:10.1016/j.cmet.2010.05.003)

Li F, Luo Z, Huang W, Lu Q, Wilcox CS, Jose PA \& Chen S 2007 Response gene to complement 32, a novel regulator for transforming growth factor- $\beta$-induced smooth muscle differentiation of neural crest cells. Journal of Biological Chemistry 282 10133-10137. (doi:10.1074/jbc. C600225200)

Li Z, Xie WB, Escano CS, Asico LD, Xie Q, Jose PA \& Chen SY $2011 a$ Response gene to complement 32 is essential for fibroblast activation in renal fibrosis. Journal of Biological Chemistry 286 41323-41330. (doi:10.1074/jbc.M111.259184)

Li L, Fan D, Wang C, Wang JY, Cui XB, Wu D, Zhou Y \& Wu LL $2011 b$ Angiotensin II increases periostin expression via Ras/p38 MAPK/CREB and ERK1/2/TGF- $\beta 1$ pathways in cardiac fibroblasts. Cardiovascular Research 91 80-89. (doi:10.1093/cvr/cvr067)
Liang H \& Ward WF 2006 PGC-1 $\alpha$ : a key regulator of energy metabolism. Advances in Physiology Education 30 145-151. (doi:10.1152/advan. 00052.2006)

Mori MA, Liu M, Bezy O, Almind K, Shapiro H, Kasif S \& Kahn CR 2010 A systems biology approach identifies inflammatory abnormalities between mouse strains prior to development of metabolic disease. Diabetes 59 2960-2971. (doi:10.2337/db10-0367)

Rakhshandehroo M, Knoch B, Muller M \& Kersten S 2010 Peroxisome proliferator-activated receptor $\alpha$ target genes. PPAR Research 2010 (doi:10.1155/2010/612089)

Schweiger M, Schreiber R, Haemmerle G, Lass A, Fledelius C, Jacobsen P Tornqvist H, Zechner R \& Zimmermann R 2006 Adipose triglyceride lipase and hormone-sensitive lipase are the major enzymes in adipose tissue triacylglycerol catabolism. Journal of Biological Chemistry $\mathbf{2 8 1}$ 40236-40241. (doi:10.1074/jbc.M608048200)

Seale P, Conroe HM, Estall J, Kajimura S, Frontini A, Ishibashi J, Cohen P, Cinti S \& Spiegelman BM 2011 Prdm16 determines the thermogenic program of subcutaneous white adipose tissue in mice. Journal of Clinical Investigation 121 96-105. (doi:10.1172/JCI44271)

Shoelson SE, Lee J \& Goldfine AB 2006 Inflammation and insulin resistance. Journal of Clinical Investigation 116 1793-1801. (doi:10.1172/JCI29069)

Uysal KT, Wiesbrock SM, Marino MW \& Hotamisligil GS 1997 Protection from obesity-induced insulin resistance in mice lacking TNF- $\alpha$ function. Nature 389 610-614. (doi:10.1038/39335)

Vlaicu SI, Tegla CA, Cudrici CD, Fosbrink M, Nguyen V, Azimzadeh P, Rus V, Chen H, Mircea PA, Shamsuddin A et al. 2010 Epigenetic modifications induced by RGC-32 in colon cancer. Experimental and Molecular Pathology 88 67-76. (doi:10.1016/j.yexmp.2009.10.010)

Wang JN, Shi N, Xie WB, Guo X \& Chen SY 2011 Response gene to complement 32 promotes vascular lesion formation through stimulation of smooth muscle cell proliferation and migration. Arteriosclerosis, Thrombosis, and Vascular Biology 31 e19-e26. (doi:10.1161/ATVBAHA.111.230706)

Winzell MS \& Ahren B 2004 The high-fat diet-fed mouse: a model for studying mechanisms and treatment of impaired glucose tolerance and type 2 diabetes. Diabetes 53(Suppl 3) S215-S219. (doi:10.2337/ diabetes.53.suppl_3.S215)

Xu H, Barnes GT, Yang Q, Tan G, Yang D, Chou CJ, Sole J, Nichols A, Ross JS, Tartaglia LA et al. 2003 Chronic inflammation in fat plays a crucial role in the development of obesity-related insulin resistance. Journal of Clinical Investigation 112 1821-1830. (doi:10.1172/JCI200319451)

Received in final form 17 October 2014

Accepted 10 November 2014

Accepted Preprint published online 10 November 2014
(C) 2015 Society for Endocrinology Printed in Great Britain 